\title{
Jet stream wind power as a renewable energy resource: little power, big impacts
}

\author{
L. M. Miller ${ }^{1,2}$, F. Gans ${ }^{1}$, and A. Kleidon ${ }^{1}$ \\ ${ }^{1}$ Max Planck Institute for Biogeochemistry, Jena, Germany \\ ${ }^{2}$ International Max Planck Research School for Earth System Modeling, Hamburg, Germany \\ Received: 27 May 2011 - Published in Earth Syst. Dynam. Discuss.: 17 June 2011 \\ Revised: 1 November 2011 - Accepted: 18 November 2011 - Published: 29 November 2011
}

\begin{abstract}
Jet streams are regions of sustained high wind speeds in the upper atmosphere and are seen by some as a substantial renewable energy resource. However, jet streams are nearly geostrophic flow, that is, they result from the balance between the pressure gradient and Coriolis force in the near absence of friction. Therefore, jet stream motion is associated with very small generation rates of kinetic energy to maintain the high wind velocities, and it is this generation rate that will ultimately limit the potential use of jet streams as a renewable energy resource. Here we estimate the maximum limit of jet stream wind power by considering extraction of kinetic energy as a term in the free energy balance of kinetic energy that describes the generation, depletion, and extraction of kinetic energy. We use this balance as the basis to quantify the maximum limit of how much kinetic energy can be extracted sustainably from the jet streams of the global atmosphere as well as the potential climatic impacts of its use. We first use a simple thought experiment of geostrophic flow to demonstrate why the high wind velocities of the jet streams are not associated with a high potential for renewable energy generation. We then use an atmospheric general circulation model to estimate that the maximum sustainable extraction from jet streams of the global atmosphere is about 7.5 TW. This estimate is about 200-times less than previous estimates and is due to the fact that the common expression for instantaneous wind power $\frac{1}{2} \rho v^{3}$ merely characterizes the transport of kinetic energy by the flow, but not the generation rate of kinetic energy. We also find that when
\end{abstract}

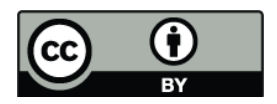

Correspondence to: L. M. Miller (lmiller@bgc-jena.mpg.de) maximum wind power is extracted from the jet streams, it results in significant climatic impacts due to a substantial increase of heat transport across the jet streams in the upper atmosphere. This results in upper atmospheric temperature differences of $>20^{\circ} \mathrm{C}$, greater atmospheric stability, substantial reduction in synoptic activity, and substantial differences in surface climate. We conclude that jet stream wind power does not have the potential to become a significant source of renewable energy.

\section{Introduction}

Energy options without carbon dioxide emissions and associated climatic impacts are necessary to avoid the current predictions of global climate change (IPCC, 2008). Renewable energy sources are seen as such options, in particular, the use of naturally generated wind power of the atmosphere by wind turbines. Surface-based wind turbine installations have proven themselves to be economically attractive examples of a renewable energy technology with tremendous growth projected for the future (American Wind Energy Association, 2007; United States Department of Energy, 2008; EEA, 2009; EWEA, 2009). Yet wind power is not necessarily limited to the atmospheric region near the surface. Strong winds in the upper atmosphere, concentrated into so-called jet streams at $7-16 \mathrm{~km}$ altitude with velocities exceeding 50 knots (or about $25 \mathrm{~m} \mathrm{~s}^{-1}$, American Meteorological Society, 1999), are seen by some as particularly rich sources of renewable wind power (Roberts et al., 2007; Vance, 2009; Archer and Caldeira, 2009). Archer and Caldeira (2009) estimated the potential of jet stream wind power as “...roughly

Published by Copernicus Publications on behalf of the European Geosciences Union. 
100 times the global energy demand". If we take the present global energy demand of 17 TW of 2010 (EIA, 2010), then this estimate would imply that $\approx 1700 \mathrm{TW}$ of wind power can be sustainably extracted from jet streams. However, this estimate is almost twice the value of the total wind power of $\approx 900$ TW (Lorenz, 1955; Li et al., 2007; Kleidon et al., 2003; Kleidon, 2010) that is associated with all winds within the global atmosphere.

Here we resolve this contradiction between the energy that can maximally extracted from the jet stream and the total power involved in generating all winds within the atmosphere. We start from the free energy balance that describes motion in the jet stream and accounts for the generation of kinetic energy, its dissipation, and the potential extraction of kinetic energy by wind turbines (see also Gans et al., 2010). With this approach, we provide a more realistic upper limit for high altitude wind power that is consistent with atmospheric energetics. The contradiction originates from the erroneous assumption that the high wind speeds of the jet streams result from a strong power source. It is well known in meteorology that jet streams reflect quasi-geostrophic flow, that is, the high wind speeds result from the near absence of friction and not from a strong power source. To demonstrate this quantitatively, we first explore the physics of jet streams, the power involved in maintaining the flow, and how these aspects change when kinetic energy is extracted from the flow in the context of a thought experiment in the following section. We then describe the implementation of a kinetic energy extraction scheme for jet stream flow into an atmospheric general circulation model in Sect. 3 as well as the setup of sensitivity simulations to various strengths of extraction. We present the results of these sensitivity simulations in Sect. 4 in terms of differences in velocity and dissipation rates, the limit on how much kinetic energy can maximally be extracted, as well as the climatic impacts that would result from a maximum extraction. In the discussion (Sect. 5), we compare our results to previous studies. In particular, we use the GCM simulations to elaborate further on the difference between the transport of kinetic energy (which is often used as a proxy for instantaneous wind power in the renewable energy literature) and the kinetic energy that can be extracted from jet streams sustainably. We close with a brief summary and conclusion.

\section{A jet stream thought experiment: why is instantaneous wind power not representative of sustainable extraction?}

To understand the relationship between wind speed and wind power in the jet stream, extractable wind power, and climatic impacts, we use a simple model of the jet stream based on basic physics (Peixoto and Oort, 1992). We will use this model to understand the dynamics of how a jet stream should be expected to respond to an additional drag to its natural flow. It a) geostrophic balance

b) quasi-geostrophic balance
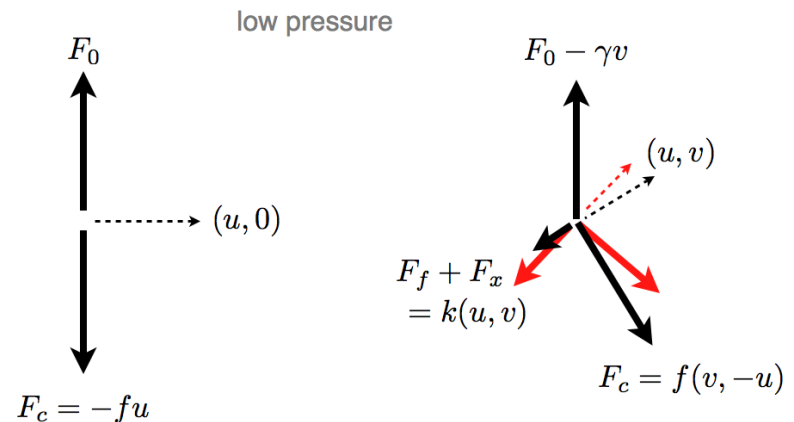

high pressure

Fig. 1. The balance of forces that describe the velocity $v=(u, v)$ of the jet stream in (a) the geostrophic balance (pressure gradient force $F_{0}$, Coriolis force $F_{\mathrm{c}}$, with Coriolis parameter $f$ ), and (b) the quasi-geostrophic balance that considers friction $F_{f}$ and removal of kinetic energy $F_{x}$ (with $F_{f}+F_{x}=k \boldsymbol{v}$ ) as well as the depletion of the pressure gradient by the zonal flow $(0,-\gamma v)$. When wind turbines extract kinetic energy from the jet stream, then the balance is shifted further away from the geostrophic balance, as indicated by the red arrows in (b).

will not be used to estimate extractable jet stream wind power from the Earth System, but simply provides an analytical approximation to jet stream dynamics.

The velocity of a jet stream $\boldsymbol{v}$ results from a neargeostrophic balance in which the pressure gradient force $F_{0}$ is balanced by the Coriolis force $F_{\mathrm{c}}$ (Fig. 1). We represent the velocity $v$ by its zonal, eastward component $u$ and its meridional, poleward component $v$. To describe the steadystate of these two components, we consider the geostrophic balance, but also introduce a drag term $k \boldsymbol{v}$ that characterizes friction and kinetic energy extraction by turbines, and a depletion term $\gamma v$ of the pressure gradient by the zonal flow of mass associated with $v$ :

$\frac{\mathrm{d} u}{\mathrm{~d} t}=f v-k u \quad \frac{\mathrm{d} v}{\mathrm{~d} t}=-f u+\left(F_{0}-\gamma v\right)-k v$

where $f$ is the Coriolis acceleration and we assumed that the pressure gradient acts in zonal, poleward direction.

In the steady state, the analytical expressions for $u$ and $v$ are:

$u=\frac{f}{f^{2}+k^{2}+k \gamma} \cdot F_{0} \quad v=\frac{k}{f^{2}+k^{2}+k \gamma} \cdot F_{0}$

The maintenance of this flow is characterized by the free energy balance of generation, dissipation, and extraction of kinetic free energy KE (Fig. 2):

$\frac{\mathrm{dKE}}{\mathrm{d} t}=G-D_{\mathrm{n}}-P_{\mathrm{ex}}$

where $G$ is the generation of kinetic energy, $D_{\mathrm{n}}$ is the natural dissipation by momentum diffusion to regions adjacent to the 


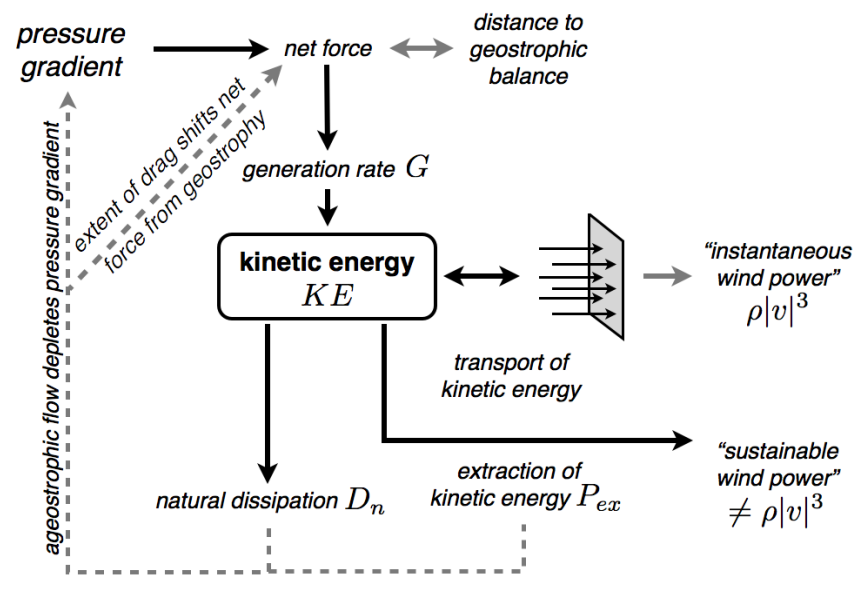

Fig. 2. The kinetic energy $\mathrm{KE}$ of the jet stream results from the balance of generation $G$, natural dissipation $D_{\mathrm{n}}$ at the edges of the jet stream due to momentum diffusion, and extraction $P_{\mathrm{ex}}$ due to the placement of wind turbines. Note that in geostrophic balance, $G=D_{\mathrm{n}}=0$ but $\mathrm{KE}>0$, so that the common metric of instantaneous wind power $\frac{1}{2} \rho|v|^{3}$ of the flow through some cross-sectional area perpendicular to the flow is not adequate to estimate the sustainable rate of kinetic energy extraction $P_{\text {ex }}$. To estimate this rate, one needs to implement the extraction of kinetic energy as a separate term into the kinetic energy balance and to evaluate its effect on the generation rate (as shown by the dashed lines).

jet stream, and $P_{\mathrm{ex}}$ is the extraction of kinetic energy by the wind turbines. The generation rate $G$ is given by the power $P$ associated with the net force acting on the mean flow, i.e.

$G=P=\boldsymbol{F}_{\text {net }} \cdot \boldsymbol{v}=\left(F_{0}-\gamma v\right) v=k \cdot \frac{\left(f^{2}+k^{2}\right)}{\left(f^{2}+k^{2}+k \gamma\right)^{2}} \cdot F_{0}^{2}$

The free energy of the jet stream is dissipated at the edges of the jet where velocity gradients deplete the momentum of the jet stream. We express this natural dissipation rate by a typical drag-like parameterization, with a drag coefficient $k_{\mathrm{n}}$ and the square of the wind speed:

$D_{\mathrm{n}}=k_{\mathrm{n}}\left(u^{2}+v^{2}\right)=k_{\mathrm{n}} \cdot \frac{f^{2}+k^{2}}{\left(f^{2}+k^{2}+k \gamma\right)^{2}} \cdot F_{0}^{2}$

The extraction of kinetic energy by wind turbines is expressed equivalently by an additional drag characterized by an additional parameter $k_{\mathrm{ex}}$ related to the number of turbines and their characteristics:

$P_{\mathrm{ex}}=k_{\mathrm{ex}}\left(u^{2}+v^{2}\right)=k_{\mathrm{ex}} \cdot \frac{f^{2}+k^{2}}{\left(f^{2}+k^{2}+k \gamma\right)^{2}} \cdot F_{0}^{2}(6)$

Hence, the value of $k$ in the expressions above is the combination of the natural friction $k_{\mathrm{n}}$ as well as the drag from the wind turbines $k_{\mathrm{ex}}$, i.e. $k=k_{\mathrm{n}}+k_{\mathrm{ex}}$.

We now use the simple model to explore the relationship between the mean jet stream velocity and the dynamics of generation and dissipation of this flow. We first note that
Table 1. Global mean values for atmospheric dissipation and climatic parameters for the control simulation (no extraction), a medium rate of extraction (4.2 TW of kinetic energy extraction), and the peak extraction simulation $(7.5 \mathrm{TW}$ of kinetic energy extraction).

\begin{tabular}{|c|c|c|c|}
\hline parameter & control & medium & peak \\
\hline ABL diss. (TW) & 584 & 482 & 419 \\
\hline free atm. diss. (TW) & 635 & 477 & 358 \\
\hline 2-meter air temp. $\left({ }^{\circ} \mathrm{C}\right)$ & 17.7 & 17.4 & 17.2 \\
\hline large scale precip. $\left(\mathrm{mm} \mathrm{day}^{-1}\right)$ & 0.68 & 0.63 & 0.54 \\
\hline conv. precip $\left(\mathrm{mm} \mathrm{day}^{-1}\right)$ & 2.95 & 2.96 & 3.00 \\
\hline
\end{tabular}

in geostrophic balance (with the absence of friction represented by $k=0$ ), the generation of KE as well as its dissipation is zero $\left(G=D_{\mathrm{n}}=0\right)$, as is the meridional flow velocity $v=0$. The mean flow of the jets is given by $u=F_{0} / f$, that is, we have a non-zero wind speed, a large stock of kinetic energy, but no power is needed to sustain its flow. This energetic view of jet stream motion is highly relevant, as the instantaneous wind power of the flow, $\frac{1}{2} \rho v^{3}$, is often used in studies to calculate wind power estimates. Since no power is involved in sustaining geostrophic flow though, the instantaneous wind power provides no indication of how much $\mathrm{ki}$ netic energy can be extracted sustainably from the flow!

As soon as we consider some drag in this balance, either by the natural dissipation of kinetic energy at the edges of the jet stream or by placing wind turbines into the flow (i.e. $k>0$ ), then the balance is shifted away from a purely geostrophic balance (Fig. 1b). In this case, the flow becomes dissipative as kinetic energy of the jet stream is dissipated by the drag, $G=D>0$ in steady state, and the flow gains a meridional component $v>0$ that depletes the driving pressure gradient (Fig. 2). The extent to which the gradient is depleted by the meridional component of the flow is captured by the parameter $\gamma$ that we introduced in the equations above.

The parameter $\gamma$ plays a pivotal role in limiting how much kinetic energy can maximally be extracted sustainably from the flow. When turbines are placed into the jet stream to extract kinetic energy to convert it further to electricity, the drag is necessarily enhanced. This results in a shift in the balance of forces further away from the geostrophic balance. Then, the generation rate $G$ may actually increase as the angle between the net force and the flow decreases, but the extent to which this increase takes place depends on how fast the driving pressure gradient is depleted by the mass transported by the zonal flow.

We demonstrate this reasoning with the simple model (Fig. 3) using the parameter values given in Appendix Table 1 . Since the value of $\gamma$ is highly uncertain, we use different values that span two orders of magnitude to evaluate its relevance. Note that a higher value of $\gamma$ implies a stronger depletion of the pressure gradient. 

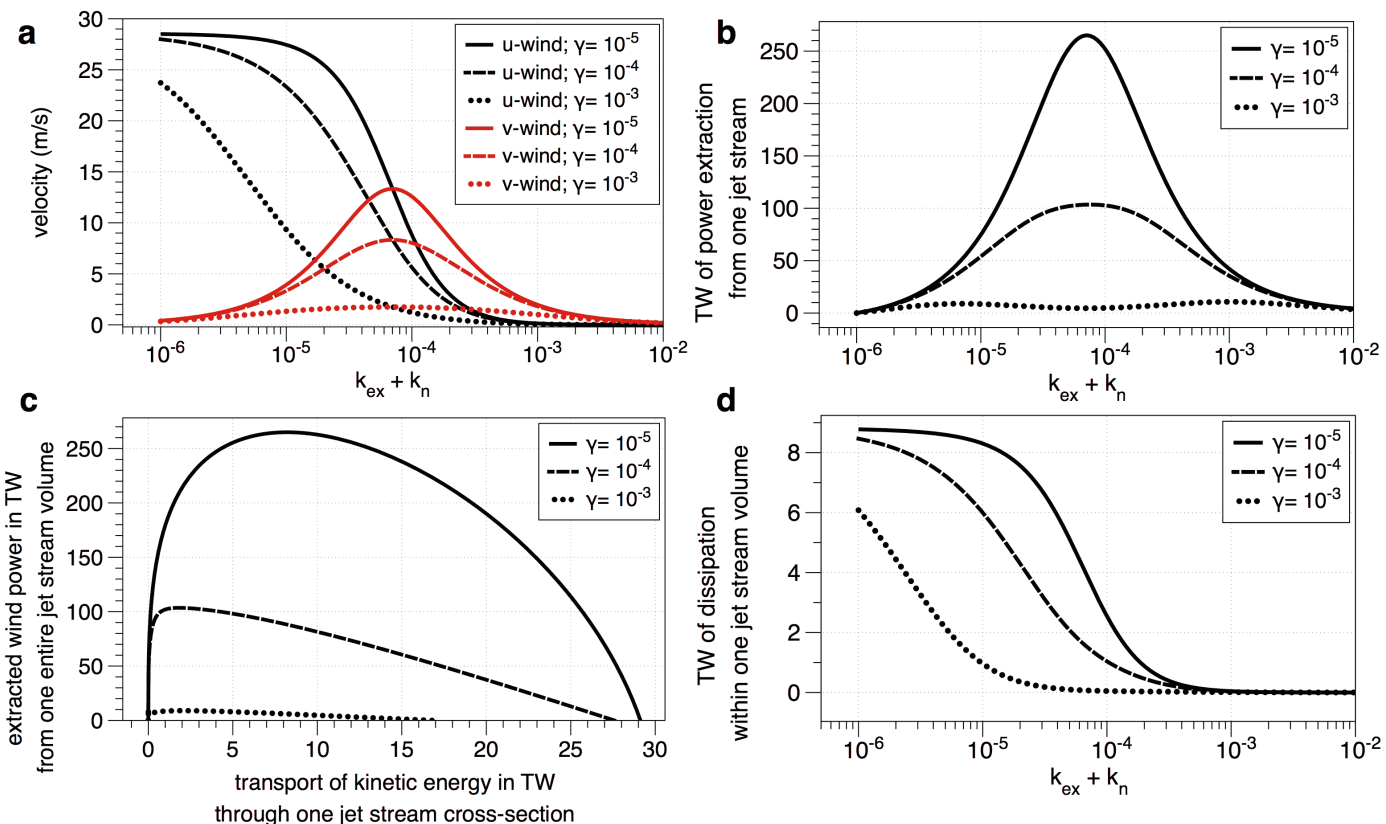

Fig. 3. Sensitivity of jet stream dynamics to the intensity of kinetic energy extraction of the simple model for different values of the intensity $\gamma$ by which the pressure gradient force is depleted. Shown are: (a) $u$ and $v$ components of the flow; (b) kinetic energy extracted from the flow due to the additional drag $k_{\mathrm{ex}}$; (c) the sustainable extraction rate $P_{\mathrm{ex}}$ of kinetic energy versus the transport of kinetic energy through a single jet stream cross-section (which is often taken as a measure of wind power); and (d) natural dissipation $D_{\mathrm{n}}$ of one jet stream. All parameters are specified in Appendix Table 1.

The first plot (Fig. 3a) shows a decrease of the $u$ component and an increase in the $v$ component with increasing values for the total drag, as would be expected by considering the three terms of the near-geostrophic balance. After reaching a peak value, the $v$ component declines for high values of the drag due to the accelerated depletion of the driving pressure gradient. The sensitivity of the decline in $u$ to the applied drag is greater and the peak in $v$ is at a lower value with greater values of $\gamma$, although the value of the drag at which the $v$ component peaks is not affected.

Figure $3 \mathrm{~b}$ shows the extracted kinetic energy as a function of the drag. The extracted power $P_{\text {ex }}$ reaches a maximum at the value of the drag at which the $v$ component is at a maximum as well, although the value of the peak is strongly affected by the value of $\gamma$. Further note that the peak of extraction occurs at a drag at which the velocity is still greater than zero, which implies that not only can all of the kinetic energy not be captured but is also limited to a maximum extraction rate.

The common expression for instantaneous wind power $\frac{1}{2} \rho|v|^{3}$ is compared to the actual rate of extraction $P_{\text {ex }}$ in Fig. 3c. What this shows is that there is no simple, linear relationship between these two properties, so that the expression of instantaneous wind power does not adequately capture the potential for wind power extraction.
Figure $3 \mathrm{~d}$ shows the decline of natural dissipation $D_{\mathrm{n}}$ with increased drag. The lines essentially track the decline of velocity as shown in Fig. 3 a.

To briefly summarize the insights gained from the geostrophic balance, we note that (i) instantaneous wind power $\frac{1}{2} \rho|v|^{3}$ provides no adequate estimate of sustainable extraction rates of kinetic energy; (ii) the maximum rate of kinetic energy extraction is constrained to less than the generation rate of kinetic energy; and (iii) the depletion rate of the pressure gradient $\gamma$ in the upper atmosphere is critical to estimate how much kinetic energy can be at maximum extracted sustainably from jet stream flow.

\section{Methods}

In order to adequately estimate the maximum rate of wind power extraction from the jet streams in the global atmosphere, we resort to a general circulation model of the atmosphere. Despite the possible limitations that such numerical models may have, this tool is critical to estimating the upper bound as it explicitly simulates the generation and dissipation of kinetic energy of jet streams in their atmospheric setting and it can explicitly simulate the effect that kinetic energy extraction from the jet streams has on the overall atmospheric dynamics. That is, the effect of the pressure gradient 
Table A1. Variables and parameters used to understand the dynamics of the jet stream (Eqs. 1-6) are listed below. Values listed in column 3 are taken from Physics of Climate by Peixoto and Oort (1992). Column 4 shows the parameters derived or estimated by the authors.

\begin{tabular}{|c|c|c|c|c|}
\hline variable & thought experiment simple model definition & Peixoto and Oort (1992) & estimates & units \\
\hline$f$ & coriolis acceleration at $30^{\circ}$ & $0.7 \times 10^{-4}$ & - & $1 \mathrm{~s}^{-1}$ \\
\hline$k_{\mathrm{n}}$ & natural jet stream drag & $10^{-6}$ & - & $1 \mathrm{~s}^{-1}$ \\
\hline$k_{\mathrm{ex}}$ & human-induced jet stream drag & - & - & $1 s^{-1}$ \\
\hline$k$ & natural and human-induced jet stream drag & - & - & $1 s^{-1}$ \\
\hline$v$ & mean control jet stream poleward (north-south) component & - & 28.6 & $\mathrm{~m} \mathrm{~s}^{-1}$ \\
\hline$u$ & mean control jet stream meridional (east-west) component & - & 0.41 & $\mathrm{~m} \mathrm{~s}^{-1}$ \\
\hline$v$ & mean control jet stream velocity & - & 28.6 & $\mathrm{~m} \mathrm{~s}^{-1}$ \\
\hline$F_{0}$ & pressure driven acceleration of $v$-component & $2 \times 10^{-3}$ & - & $\mathrm{ms}^{-2}$ \\
\hline$F_{\mathrm{c}}$ & Coriolis force & - & - & $\mathrm{kg} \mathrm{ms} \mathrm{s}^{-2}$ \\
\hline$F_{\mathrm{f}}$ & frictional force & - & - & $\mathrm{kg} \mathrm{ms} \mathrm{s}^{-2}$ \\
\hline$F_{x}$ & removal of kinetic energy & - & - & $\mathrm{kg} \mathrm{m} \mathrm{s}^{-2}$ \\
\hline$F_{\text {net }}$ & frictional force and KE removal force $\left(F_{\mathrm{f}}+F_{x}=k \boldsymbol{v}\right)$ & - & - & $\mathrm{kg} \mathrm{m} \mathrm{s}^{-2}$ \\
\hline $\mathrm{KE}$ & jet stream kinetic energy & - & - & $\mathrm{kg} \mathrm{ms} \mathrm{s}^{-2}$ \\
\hline$P$ & power associated with the net force $\left(F_{\text {net }}\right)$ & - & - & $\mathrm{kg} \mathrm{m}^{2} \mathrm{~s}^{-3}$ \\
\hline$G$ & generation rate of jet stream kinetic energy & - & - & $\mathrm{kg} \mathrm{m}^{2} \mathrm{~s}^{-3}$ \\
\hline$\gamma$ & depletion rate of the jet stream gradient & - & - & $1 \mathrm{~s}^{-1}$ \\
\hline$D_{\mathrm{n}}$ & natural wind dissipation by mom. diffusion & $10^{-4}$ & - & $\mathrm{kg} \times \mathrm{m}^{2} \mathrm{~s}^{-3}$ \\
\hline$\Delta z$ & vertical extent of one jet stream & - & $10^{3}$ & $\mathrm{~m}$ \\
\hline$\Delta R \phi$ & horizontal extent of one jet stream & - & $10^{6}$ & $\mathrm{~m}$ \\
\hline$L$ & length of one jet stream at $30^{\circ}$ and $10 \mathrm{~km}$ altitude & - & $3.4 \times 10^{7}$ & $\mathrm{~m}$ \\
\hline$\rho$ & density of one jet stream at $10 \mathrm{~km}$ altitude & - & 0.4 & $\mathrm{~kg} \mathrm{~m}^{-3}$ \\
\hline$V$ & volume of one jet stream at $30^{\circ}$ and $10 \mathrm{~km}$ altitude & - & $2.7 \times 10^{16}$ & $\mathrm{~m}^{3}$ \\
\hline$\rho \cdot V$ & mass of one jet stream at $30^{\circ}$ and $10 \mathrm{~km}$ altitude & - & $10^{16}$ & $\mathrm{~kg}$ \\
\hline
\end{tabular}

depletion that was captured by $\gamma$ in the simple model above is explicitly simulated.

In this study, we use PlaSim, an atmospheric general circulation model of intermediate complexity (Fraedrich et al., 2005; Lunkeit et al., 2007) to quantify wind power extraction from the jet streams. We use this model with T42 spectral resolution, corresponding to a horizontal resolution of about $2.8^{\circ}$ longitude by $2.8^{\circ}$ latitude, ten atmospheric layers, a mixed-layer ocean model with prescribed oceanic heat transport, interactive sea-ice model, a simple land surface model, and prescribed ice sheets. Previous research shows that this model adequately captures the present-day climate and key sensitivities (Fraedrich et al., 2005; Kleidon et al., 2006).

In the following, we first describe briefly how we extract kinetic energy from the jet stream and then describe the setup of the sensitivity simulations.

\subsection{Kinetic energy extraction from jet streams within a GCM}

To simulate kinetic energy extraction, we apply an additional momentum flux $J_{\text {turbines }}$ to the vertical diffusion scheme for momentum for those grid cells at which the velocity is greater than a threshold wind speed of $v_{\text {jet }}$ at a given model time step. Vertical diffusion of momentum represents nonresolved turbulent exchange between layers and is applied to the horizontal wind components, as well as to potential temperature and specific humidity. The momentum fluxes $J_{u, v}$ for the $u$ and $v$ wind components are expressed as:

$J_{u, v}=\rho K_{\mathrm{m}} \frac{\partial(u, v)}{\partial z}$

where $\rho$ is the air density, $K_{\mathrm{m}}$ is the exchange coefficient for momentum (which depends on stability, among other factors), and $z$ is the vertical coordinate. The change in wind speed $\partial(u, v) / \partial t$ by momentum diffusion is then given by a diffusion equation:

$\frac{\partial(u, v)}{\partial t}=\frac{1}{\rho} \frac{\partial J_{(u, v)}}{\partial z}-\frac{1}{\rho} J_{\text {turbines }}$

where $J_{\text {turbines }}$ is the additional drag exerted by the wind turbines. The additional drag is in turn expressed in a similar way as the surface drag as:

$J_{\text {turbine }}=\rho\left(C_{\mathrm{ex}}|\boldsymbol{v}|\right) \cdot \boldsymbol{v}$

where $C_{\mathrm{ex}}$ is the representative drag coefficient corresponding to the momentum extraction by wind turbines of a certain intensity. 
We then diagnose the extracted power $P_{\text {ex }}$ by turbines by

$P_{\text {ex }}=J_{\text {turbine }} \cdot \boldsymbol{v}$

as well as the frictional dissipation $D_{\mathrm{n}}$ by

$D_{\mathrm{n}}=\frac{\partial J_{(u, v)}}{\partial z} \cdot(u, v)$

\subsection{Simulation setup and analysis}

A sensitivity analysis with 15 simulations was performed with different values of $C_{\mathrm{ex}}=\left[10^{-10}: 10^{-4}\right]$ was applied to all grid points within the model grid with wind velocities that exceeded $v_{\text {jet }}>25 \mathrm{~m} \mathrm{~s}^{-1}$. These simulations were compared to the "control" simulation of the present-day without momentum extraction $\left(C_{\mathrm{ex}}=0\right)$. The simulation at which extracted wind power is at maximum will be referred to as "peak extraction" in the following. Through this setup, we establish the natural, upper limit of extractable wind power from high altitude jet streams without including methodological, technological, or engineering considerations. All simulations were run for $30 \mathrm{yr}$ with the first $10 \mathrm{yr}$ discarded to exclude spin-up effects.

The simulations were evaluated with respect to the resulting rate of kinetic energy extraction $P_{\text {ex }}$ as well as variables of the jet stream, such as the $u$ and $v$ component as in the simple model above. In addition, we investigated the impacts of kinetic energy extraction on the dynamics of the jet streams and the climate system in general. To do so, we evaluated the differences in the the 20 -yr means for the "control" and "peak extraction" simulations in terms of the $u$-wind, $v$-wind, 2-m air temperature, large-scale precipitation, convective precipitation, incoming solar radiation at the top of the atmosphere, and outgoing longwave radiation at the top of the atmosphere. To quantify changes in synoptic activity, the difference in standard deviation of simulated surface pressures (daily mean) for the 20 -yr simulation dataset are compared.

Additional sensitivity analyses were performed with thresholds of $v_{\text {jet }}>20 \mathrm{~m} \mathrm{~s}^{-1}$ and $v_{\text {jet }}>15 \mathrm{~m} \mathrm{~s}^{-1}$ to evaluate the sensitivity of this threshold on the jet stream wind power estimates.

\section{Results}

\subsection{Maximum extractable wind power and energetics of jet streams}

The sensitivity of the extractable wind power to the drag $C_{\mathrm{ex}}$ associated with wind turbines is shown in Fig. 4. As in the case of the simple thought experiment, it shows a pronounced peak at intermediate values of $C_{\mathrm{ex}}$. The peak extractable power in these simulations is $7.5 \mathrm{TW}$ of mechanical power removed from the flow. Because of wake turbulence behind the turbines (which is not included in the simulations), not all

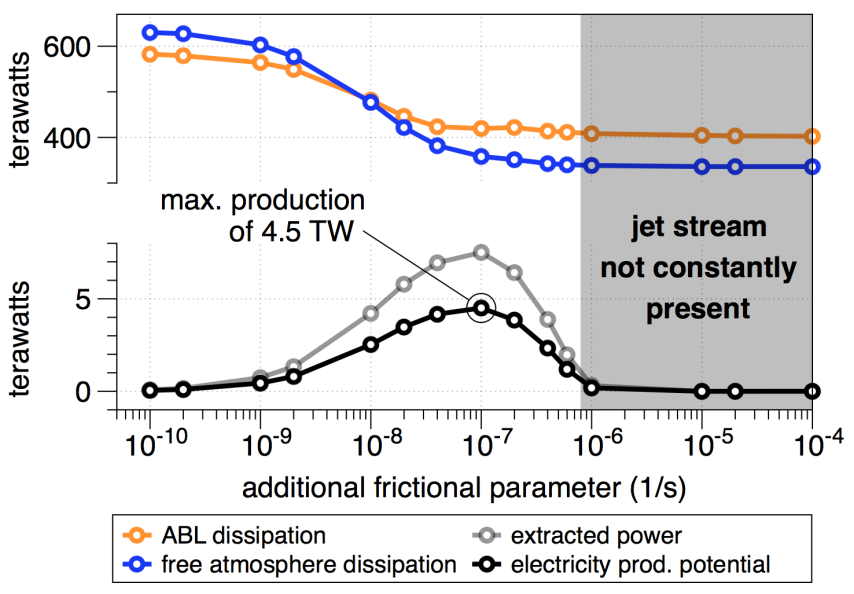

Fig. 4. Sensitivity of extracted kinetic energy from jet streams $P_{\mathrm{ex}}$ and total atmospheric dissipation $D_{\mathrm{n}}$ to the additional drag $C_{\mathrm{ex}}$ imposed by wind turbines.

of this extracted power is likely to be available as mechanical power to drive the turbine. Previous research showed a maximum, but unachievable, conversion efficiency from extracted power to mechanical power of $59.3 \%$ (Lanchester, 1915; Betz, 1920; Garrett and Cummins, 2007). Assuming a $60 \%$ conversion efficiency to mechanical turbine power and $100 \%$ conversion efficiency from mechanical to electrical power, this upper bound yields a peak potential for electricity production of $4.5 \mathrm{TW}$.

This upper estimate of extractable wind power is relatively insensitive to the threshold velocity $v_{\text {jet }}$. In sensitivity simulations with $v_{\text {jet }}=20 \mathrm{~m} \mathrm{~s}^{-1}$, the peak extracted power drops from $7.5 \mathrm{TW}$ to $7.2 \mathrm{TW}$. When $v_{\text {jet }}=15 \mathrm{~m} \mathrm{~s}^{-1}$ is used, the peak drops further to $6.7 \mathrm{TW}$. The low value of extractable power in the climate model simulations suggests a high value of $\gamma$ of at least $\gamma=10^{-3}$ in the simple model, that is, that kinetic energy extraction results in a strong depletion of the upper atmospheric pressure gradient.

The dynamics and sensitivities as well as the maximum in extraction at intermediate values of $C_{\mathrm{ex}}$ directly correspond to the ones shown by the simple model of the previous section. The sensitivity of upper atmospheric winds and jet stream dissipation to the intensity of kinetic energy extraction from the jet streams is shown in Fig. 5. As in the case of the simple model (Fig. 3a), the zonal component of the upper atmospheric velocities decreases in response to enhanced drag, while the meridional component increases. This is accompanied with a general decrease of the total dissipation of the jet stream (Figs. 3d and 5b). Since velocities decreased considerably and below the threshold value of $v_{\text {jet }}$, jet streams were not continuously present in the simulations for drag coefficients $C_{\mathrm{ex}} \geq 10^{-6}$.

Note that in addition to the dynamics represented by the simple model, the climate model simulations show a successive decrease in the generation of kinetic energy in the global 

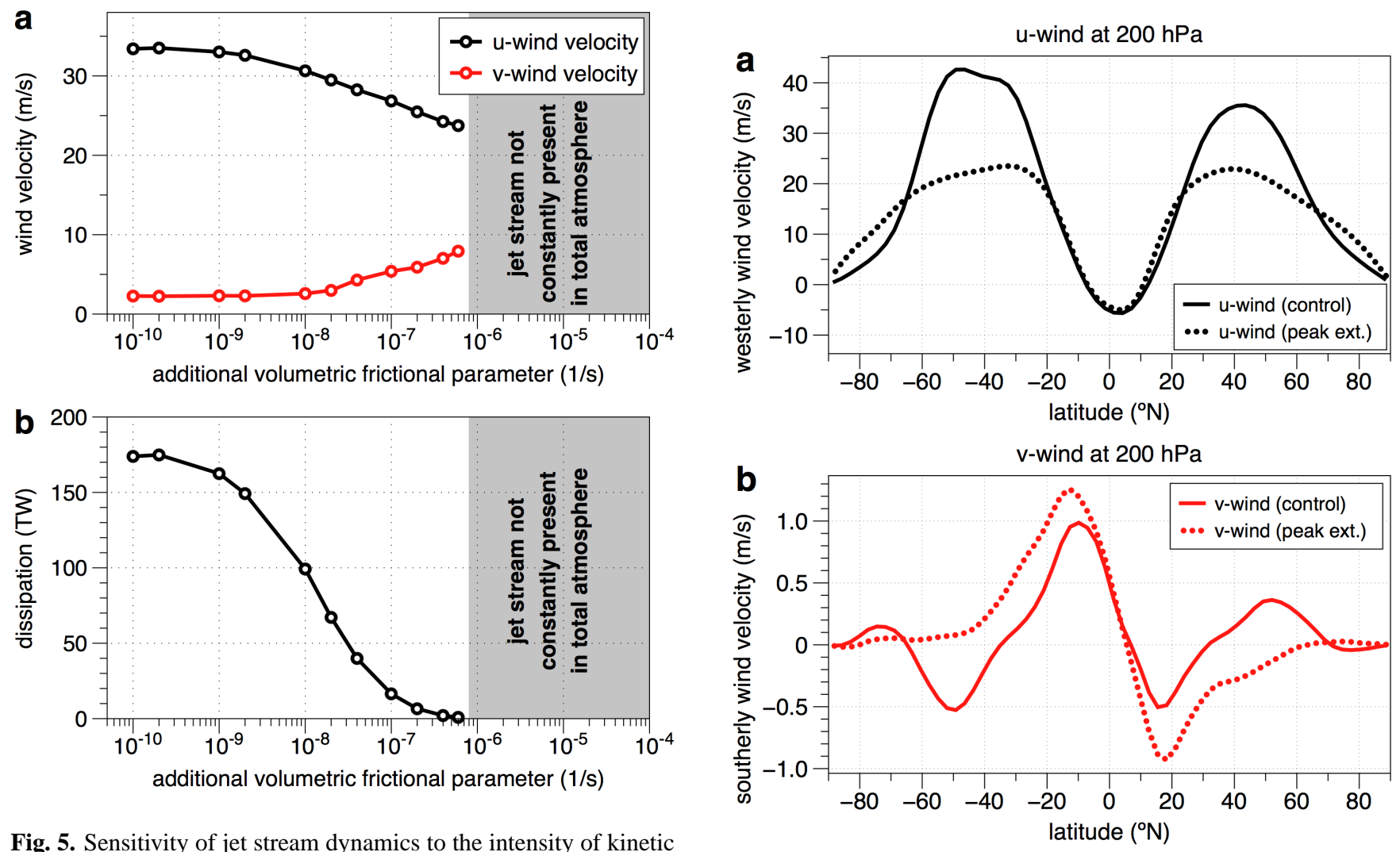

Fig. 5. Sensitivity of jet stream dynamics to the intensity of kinetic energy extraction $C_{\mathrm{ex}}$ from jet streams with $v_{\text {jet }}=25 \mathrm{~m} \mathrm{~s}^{-1}$ in terms of (a) the mean $u$ - and $v$-wind velocities at $200 \mathrm{hPa}$ and (b) the dissipation rate within those atmospheric regions at which the wind velocity is $>25 \mathrm{~m} \mathrm{~s}^{-1}$.

atmosphere, as reflected by the reduction of dissipation in the free atmosphere and the atmospheric boundary layer (Fig. 4). This decrease in kinetic energy generation by the atmosphere is about two orders of magnitude larger than the extracted mechanical power at peak extraction. In order to understand why this is an inevitable consequence of the kinetic energy extraction from jet streams, we need to first investigate the broader climatic impacts.

\subsection{Climatic impacts from jet stream wind power extraction}

The substantial extraction of kinetic energy from jet streams has a marked global impact on atmospheric dynamics in the simulations. As already shown above, the meridional wind component increases substantially as a result of the kinetic energy extraction. This is shown in Fig. 6, where the upper atmospheric winds at peak extraction are compared to the control. Zonal flow in the mid-latitudes is reduced to about $2 / 3$ at peak extraction when compared to the control, and this reduction of zonal flow is accompanied with a substantial increase in meridional flow. This meridional flow increase is responsible for additional heat transport from the equatorial region towards the poles, as shown in Fig. 7.

Fig. 6. Zonal annual means of the wind fields at $200 \mathrm{hPa}$ for (a) the zonal $(u)$ wind component and the (b) meridional $(v)$ wind component. Shown are the control simulation (solid line) and the simulation with maximum kinetic energy extraction (dotted line).

The enhanced meridional flow in the upper atmosphere has important effects on climate and atmospheric dynamics. The enhanced meridional flow transports more heat in the upper atmosphere. This results in a substantial difference in upper atmospheric temperatures of more than $20^{\circ} \mathrm{C}$ in both high-altitude polar atmospheres (Fig. 8a). These differences occur adjacent to the regions in which the kinetic energy is extracted (Fig. 8b), substantiating the direct link between extraction and enhanced meridional heat transport in the upper atmosphere.

This strong warming in the extratropical upper atmosphere has further consequences. It results in a reduced vertical temperature gradient in the extratropics (Fig. 8a) and thereby in an enhanced vertical stability of the atmosphere. As a result, the ability of the atmosphere to generate kinetic energy is greatly reduced. This is reflected in a lower total dissipation in the free atmosphere and the atmospheric boundary layer (Fig. 4, also Table 1), with a $44 \%$ decrease in free atmosphere dissipation (635 TW to $358 \mathrm{TW}$ ) and a $29 \%$ decrease in boundary layer dissipation $(584 \mathrm{TW}$ to $419 \mathrm{TW})$, with these differences in the energetics suggesting substantial differences to the Lorenz Energy Cycle (Lorenz, 1955) although further study into the specific differences in 

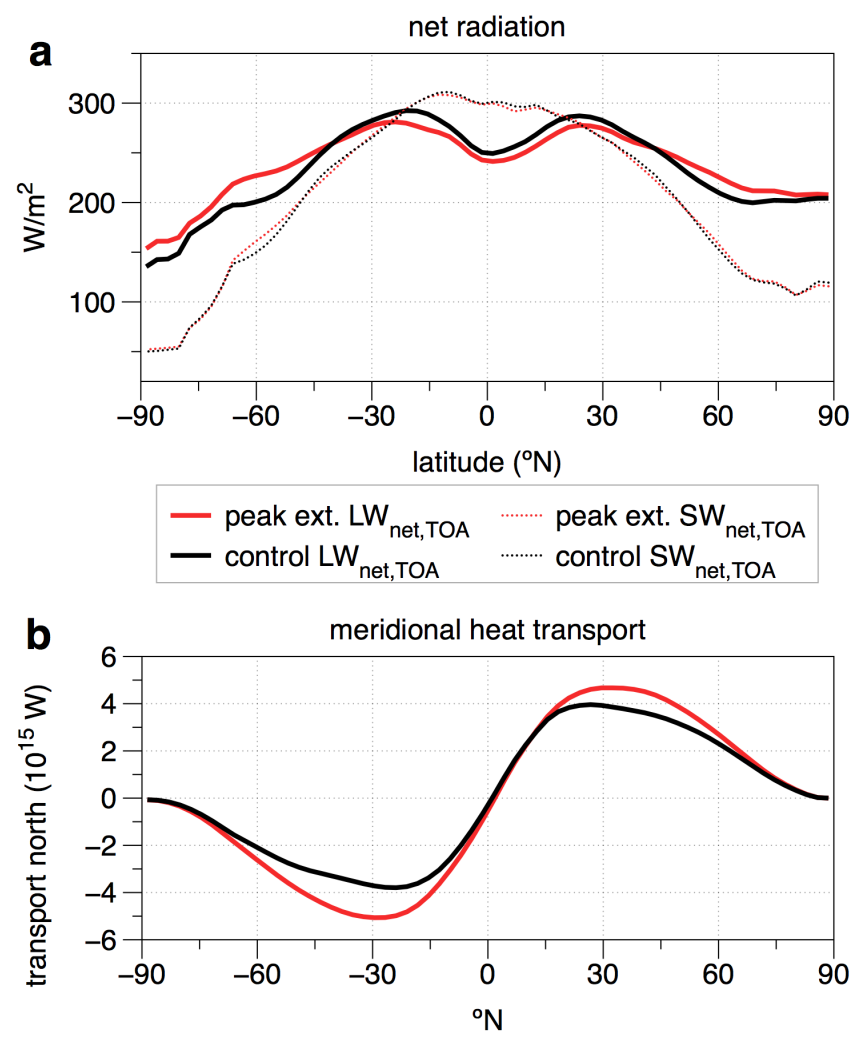

- peak extraction - control

Fig. 7. (a) Meridional profile of the zonal mean net shortwave radiation at the top of the atmosphere $\left(S W_{\text {net,TOA }}\right)$ and zonal mean net longwave emission at the top of the atmosphere ( $\left.L W_{\text {net,TOA }}\right)$ for the control and peak extraction simulations. (b) Mean heat transport of the atmosphere and oceans as implied by the top-of-atmosphere fluxes for the control and peak extraction simulations

the energy components and conversion rates is still needed. These differences do result in the reduction in kinetic energy generation, with the associated overall heat transport resulting in a greater radiative imbalance at the top of the atmosphere (Fig. 9). Even though this enhanced radiative imbalance would seem to imply a stronger radiative forcing and thereby a greater ability of the atmosphere to generate motion, it is critical to note that this radiative imbalance is rather the consequence of reduced overall motion within the atmosphere.

In the simulation of peak extraction, we find considerable differences in climate (Fig. 10). The climatic differences shown in Table 1 are not related to a mean change in radiative forcing, as would be the case for climatic change due to alterations of the atmospheric greenhouse effect, but result directly from the weakened energetics of atmospheric motion. In particular, we find that the variability of surface pressure is considerably reduced in the mid-latitudes, indicating a reduction of synoptic activity. This reduction is consistent with the general reduction of kinetic energy gener- a
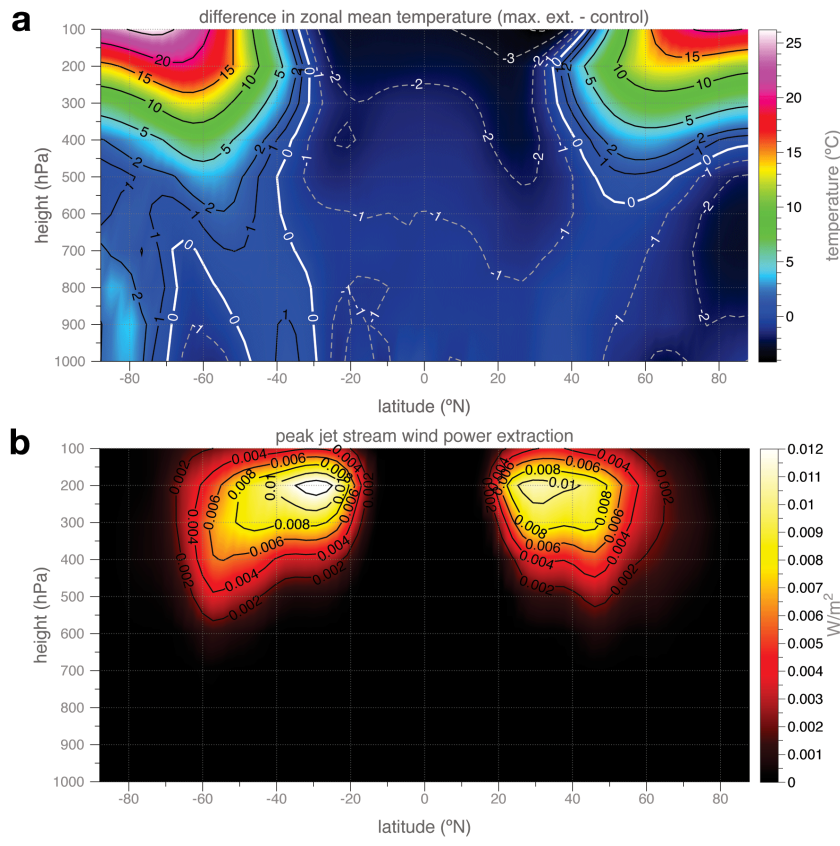

Fig. 8. (a) Difference in the zonal mean temperature. (b) The maximum extracted wind power for the $20 \mathrm{yr}$ mean is mainly derived from the southern hemisphere at a height of $200 \mathrm{hPa}(\approx 10 \mathrm{~km})$.

ation within the atmosphere. The associated differences in mean $2 \mathrm{~m}$ air temperature in the mid-latitudes are consistent with this reduced synoptic activity, with pronounced cooler temperatures over land.

\section{Discussion}

Our estimate of maximally extractable wind power from jet streams of $7.5 \mathrm{TW}$ is substantially lower than the estimate of 1700 TW by Archer and Caldeira (2009). Naturally, there must be a simple reason why our estimate is so much lower. In the following, we first describe that our results are consistent with the basic physics of jet streams. Hence, the difference in estimates likely originates from the difference in methodology. We relate this difference to the flaw in the common methodology that derives extractable wind power from wind speeds rather than from the free energy balance that describes the generation, dissipation, and extraction of kinetic energy (e.g. Gans et al., 2010 gives a detailed description of the severe limitations of the common method) and illustrate this flaw with output from the climate model simulations. We then discuss the implications of these results in terms of the methodology that should be used for maximum estimates of wind power and for the prospects of wind power from high altitude winds.

First, we point out that our results are fully consistent with the basic physics that describe the dynamics of jet streams and what would be expected when jet streams are disturbed 

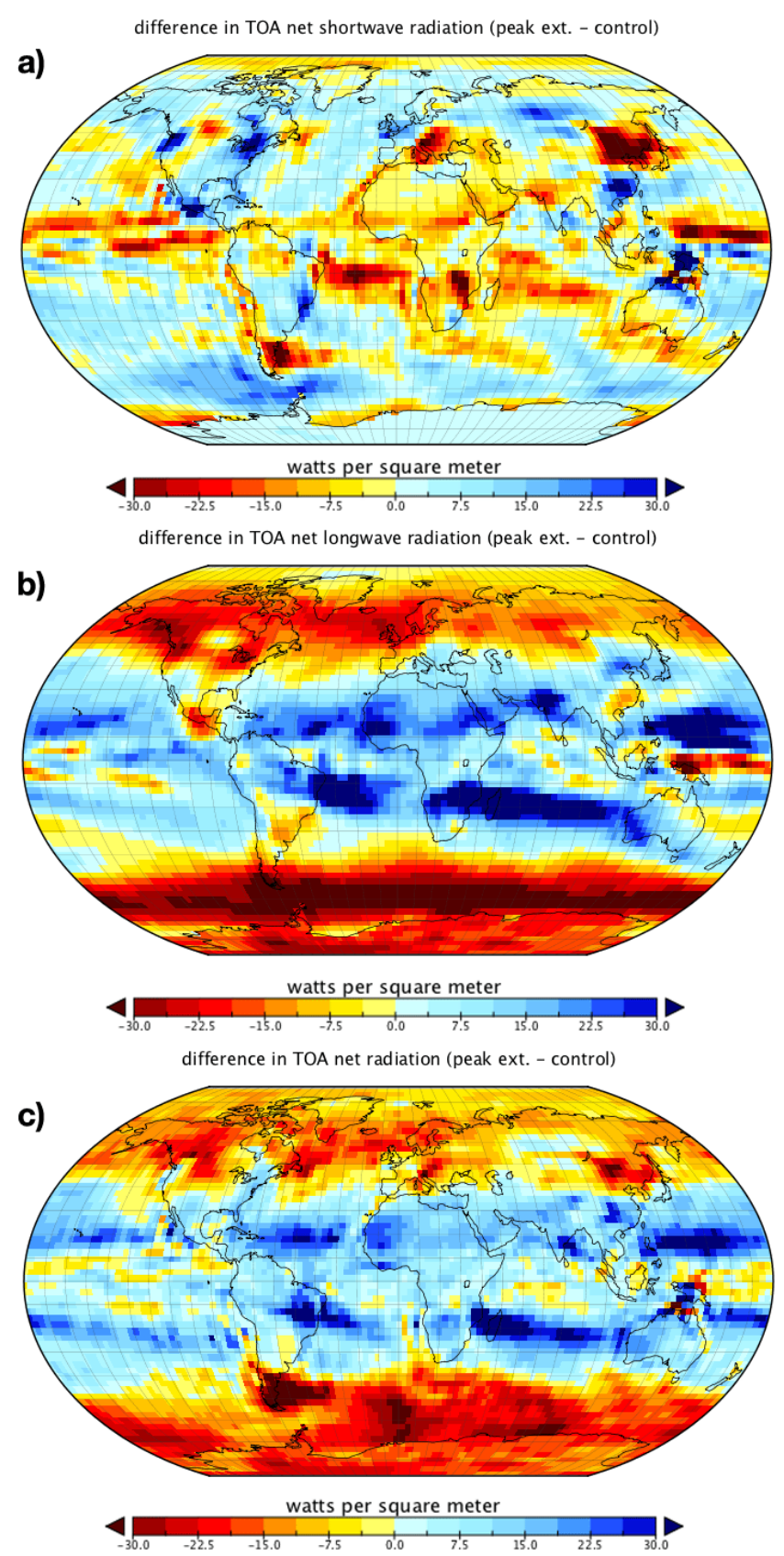

Fig. 9. Net radiation differences between the peak extraction and control simulations at the top of the atmosphere for (a) shortwave (solar) radiation, (b) longwave (thermal) radiation, and (c) net radiation. Note how the extraction of kinetic energy from the jet streams results in relatively small changes to the net shortwave radiation (a) but also corresponds to relatively large changes to the net longwave radiation (b) in response to the enhanced upper-atmospheric meridional heat transport to the poles. The overall difference at the top of the atmosphere is less net radiation in the tropics and more net radiation in the mid-latitudes and polar regions $(\mathbf{c})$. by kinetic energy extraction. As it is well known in meteorology, jet streams result from a near geostrophic balance of forces, that is, the quasi-geostrophic flow results from the near absence of friction. Hence, little power is involved in maintaining the high wind speeds of the jet streams. This near-geostrophic nature of jet streams is reflected in our low maximum estimate of extractable wind power.

The potential impacts of extraction that we find in the climate model simulations are consistent with the potential alteration of the near-geostrophic balance of forces by kinetic energy extraction of wind turbines. This balance is disturbed by an additional drag by wind turbines, and this drag is unavoidable as kinetic energy needs to be extracted from the flow to rotate the turbine. Through this additional drag, the resulting motion is brought further away from the geostrophic balance, yielding a stronger ageostrophic component of the flow. The climate model simulations show this expected change and the simulated climatic impacts result from this enhanced ageostrophic flow in the upper atmosphere. Furthermore, the climatic impacts that we find are consistent with those reported by Archer and Caldeira (2009). Specifically, Archer and Caldeira (2009) p.315 found a "... strengthening of the Equator-to-Pole thermal difference [that was] caused by the weakening of the global winds". Such a strengthening of the surface temperature difference at the surface is also found in our simulations at peak extraction and consistent with our interpretation.

Hence, the discrepancy of our estimate to previous ones should be found in the methodology. While extractable wind power is commonly determined from the wind speed by $\frac{1}{2} \rho v^{3}$, we took a different approach and considered the free energy balance of kinetic energy generation, dissipation, and extraction. In steady state, the rate of kinetic energy extraction needs to be balanced by how much kinetic energy is generated and must be less than the rate at which it is naturally transferred out of the jet stream by momentum diffusion. The mean stock of free energy, the kinetic energy of the flow $\frac{1}{2} \rho v^{2}$, then reflects not just the generation rate, but also the intensity of its natural depletion and the extent of extraction. However, as already shown in Sect. 2 above, the instantaneous wind power density of $\frac{1}{2} \rho v^{3}$ is not related to the maximum sustainable rate at which kinetic energy can be extracted from jet streams. The instantaneous wind power density merely describes the transport of kinetic energy by the flow through a cross section perpendicular to the flow, but yields little information about the generation and natural depletion rate of kinetic energy. This is in particular the case for geostrophic flow, where no generation is needed to sustain geostrophic motion because of the absence of frictional dissipation.

This critical distinction between the transport of kinetic energy in contrast to the natural rate of depletion is shown in Fig. 11 for the climate model simulation. The high rates of the mean transport of kinetic energy at $200 \mathrm{hPa}$ height shown in Fig. 11a are consistent in pattern and magnitude 

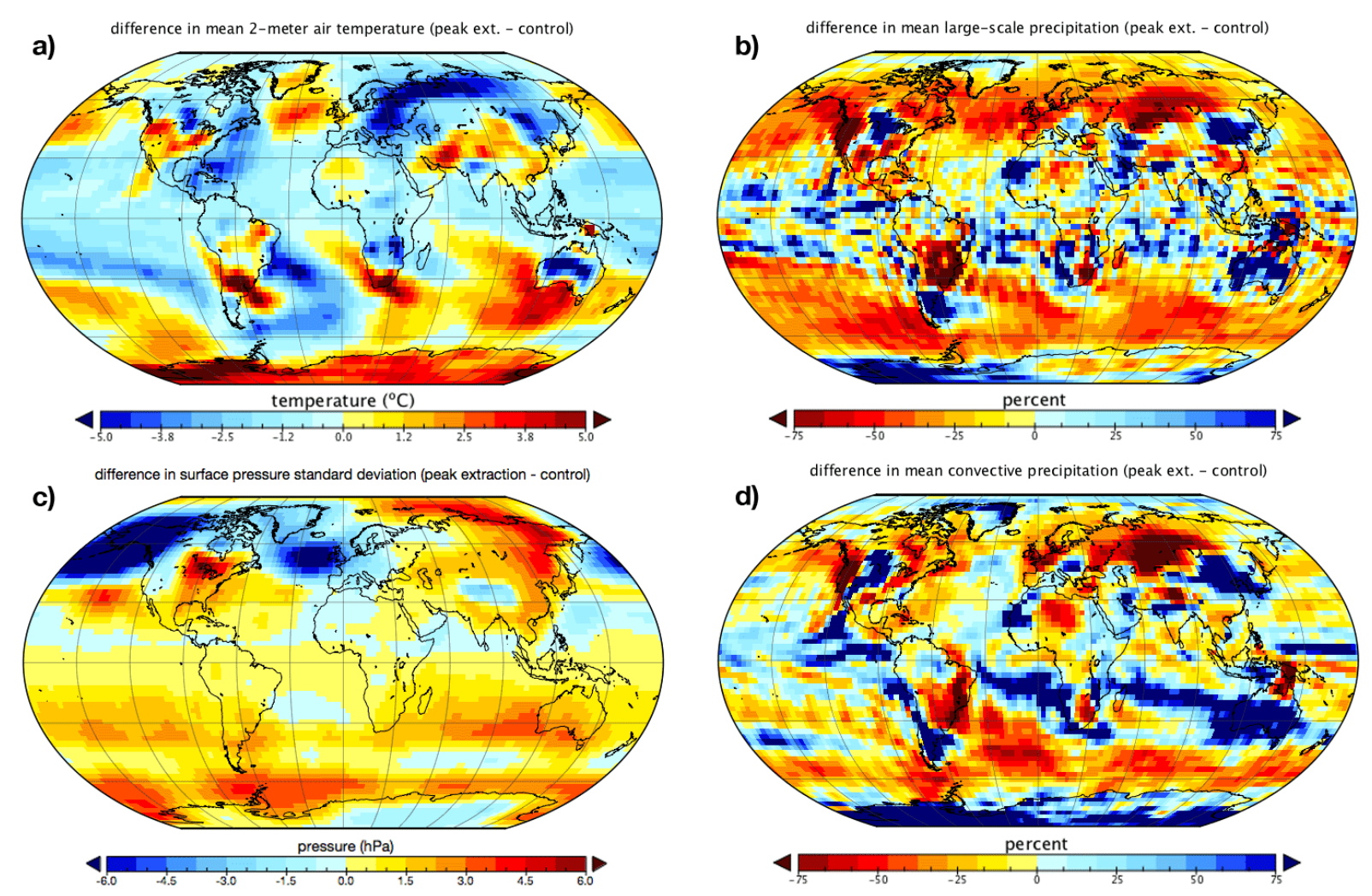

Fig. 10. Mean 20-yr simulation differences between the maximum jet stream wind power extraction and the control simulation with differences in (a) 2-m air temperature, (b) large-scale precipitation, and (c) surface pressure variability, and (d) convective precipitation shown.

with the maps shown by Archer and Caldeira (2009), although these are referred to by Archer and Caldeira (2009) as wind power density. Figure 11b shows the natural depletion rate at $200 \mathrm{hPa}$ due to momentum diffusion with no extraction. As can be seen, the natural depletion rate at $200 \mathrm{hPa}$ is 4 orders of magnitude smaller than the transport of kinetic energy. Since in the natural steady state, the generation of motion balances its depletion, it is this depletion rate that characterizes the power involved in sustaining the flow. The maximum estimates for wind power extraction are then even lower, as shown in Fig. 11c, and show relatively little correspondence to the patterns of the transport of kinetic energy. Therefore, the transport of kinetic energy by jet streams cannot be used to provide estimates of maximum sustainable rates of kinetic energy extraction.

These results have broader implications for how maximum estimates of wind power should to be computed in general. First, we showed that it is critical to consider extraction as a term in the free energy balance of kinetic energy and use this balance as the fundamental limit on maximum possible rates of kinetic energy extraction by wind turbines (following Gans et al., 2010). As wind speeds merely reflect the stock of kinetic energy within the atmosphere, these cannot be used to provide such estimates of maximum possible extraction rates. Second, our climate model results showed that there are substantial, first order effects when kinetic energy is extracted that affect the ability of the atmosphere to generate kinetic energy. To capture these effects, physically-based models that simulate the generation of kinetic energy and the effects of extraction on this generation rate are critical for estimates of upper limits of wind power, despite all the potential flaws that these models may have. Upper estimates that are based on observed wind speeds cannot represent the free energy balance of kinetic energy and the feedbacks of extraction on generation rates of kinetic energy and thereby cannot provide physically consistent estimates.

Quantifying the impact of wind power on climate as the ratio of kinetic energy generation to the energy extraction, jet stream wind power has a very high impact. Taking the global numbers from the peak extraction simulation, the atmosphere generated 778 TW (36\% less than the control) when influenced by a peak jet stream extraction rate of 7.5 TW, for an impact of $\approx 100: 1$ An impact of $\approx 20: 1$ can be derived from our previous study of near-surface wind energy extraction over all non-glaciated land, where the atmosphere generated $1065 \mathrm{TW}$ (3\% less than the control) when influenced by a peak near-surface wind energy extraction rate of 57 TW (see Fig. 5b of Miller et al., 2011). This difference in impact suggests that utilizing wind at different altitudes within the atmosphere can have substantially different effects 

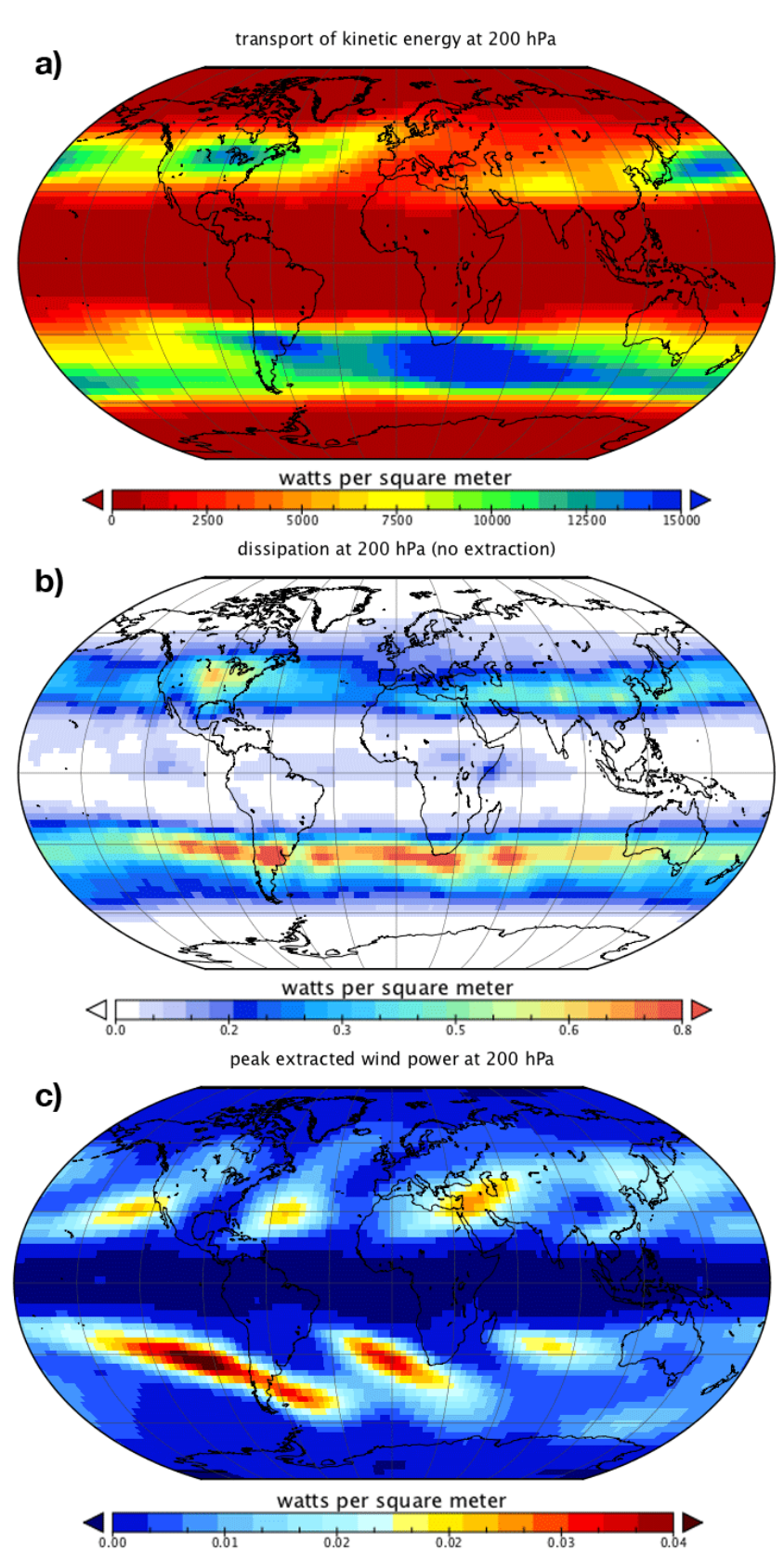

Fig. 11. (a) Mean transport of kinetic energy through a cross section at $200 \mathrm{hPa}$ derived by $\frac{1}{2} \rho \boldsymbol{v}^{\mathbf{3}}$ where $\rho$ is the air density and $\boldsymbol{v}$ is the wind velocity. (b) The mean depletion $\left(D_{\mathrm{n}}\right)$ within the $200 \mathrm{hPa}$ model layer due to momentum diffusion under control conditions and c) mean maximum extraction $\left(P_{\text {ext }}\right)$ within the $200 \mathrm{hPa}$ model layer.

on the global generation rate of kinetic energy. Directly related to this, reviewer Kirk-Davidoff (2011) suggests that to minimize the impact of higher-altitude wind power technologies, wind energy extraction should be actively researched at lower altitudes (e.g. Lansdorp and Williams, 2006) and adjacent to, but not within the jet streams. While we ten- tatively agree with this interpretation, supportive research is still required.

This study should be clearly recognizable as an extreme scenario, with the overall aim being to estimate an upperbound to jet stream kinetic energy extraction. It would seem technically nearly impossible to continuously track the regions at which wind speeds exceed $25 \mathrm{~m} \mathrm{~s}^{-1}$ and extract substantial rates of kinetic energy from the upper atmosphere at the global scale to get close to our estimate. Furthermore, substantial interference with the jet streams would change the climate substantially, in particular through the weakening of the atmospheric heat engine by two orders of magnitude more than the power gained by extraction. Hence, it would seem that jet stream wind power has very little potential to contribute to the challenge of meeting the primary energy demands of humans.

\section{Summary and conclusions}

We used a new, physically consistent method to estimate the maximum rate of kinetic energy extraction from high altitude winds of $>25 \mathrm{~m} \mathrm{~s}^{-1}$. This method represents kinetic energy extraction as a term in the free energy balance of kinetic energy generation, dissipation, and extraction. Our estimate for maximum sustainable extraction of kinetic energy from jet streams is $7.5 \mathrm{TW}$ and is about two orders of magnitude less than previous estimates. Our substantially lower estimate reflects physical consistency with the free energy balance of kinetic energy, the impacts that substantial kinetic energy extraction has on the generation rate, and is consistent with the well-established notion that jet streams represent near-geostrophic flow. In contrast, other estimates are often based on wind speeds that are then used to compute the transport of kinetic energy by the flow. This term is then misinterpreted as being the sustainable extraction rate of kinetic energy. Hence, it would seem that velocity based estimates of wind power are flawed because they cannot infer the terms of the free energy balance and the effects of extraction on the generation rate of free energy, but these terms are the ones that ultimately limit sustainable extraction rates.

We conclude that it is critical to start with the free energy balance for evaluating the potential contributions of different forms of renewable energy to the growing human needs for energy. These estimates need to be performed in an Earth system context to account for first-order consequences of extraction on the generation rates of free energy. Our application of this physically-based approach to high altitude winds shows that in practicality, there is a very limited potential of jet streams to contribute to human energy needs, and if it were used, only with a very substantial climatic consequence. Previous claims by researchers that "if you tapped into 1 percent of the power in high-altitude winds, that would be enough to continuously power all civilization" (Blackman, 2009) are based on estimates that do not account 
for the limitations imposed by the free energy balance and are therefore physically flawed. Such statements substantiate the urgent need for a physically-based approach that quantifies the dynamics of free energy generation and depletion in the context of Earth system functioning in order to understand how much these can potentially contribute to a human renewable energy future.

Acknowledgement. The authors would like to thank K. Hasselmann, D. B. Kirk-Davidoff, and V. Lucarini for their helpful reviews of this manuscript. J. C. Bergmann also noted a potentially confusing axis label in Fig. 6. Ryan Pavlick, the Biospheric Theory and Modeling Group, Ulrike Stahl, and Kerry Hinds provided helpful discussions and comments on this topic. Colleagues of the International Max Planck Research School's Advanced Scientific Writing Course, particularly Jochem Marotzke and Dallas Murphy, provided numerous constructive comments on an earlier version of this manuscript. Adam Maxell assisted with plotting.

Edited by: H. Held

\section{References}

American Meteorological Society: Glossary of Meteorology, http://amsglossary.allenpress.com/glossary/search?p=1\&query= jet+stream\&submit=Search last access: 24 May 2011, 1999.

American Wind Energy Association: 20 percent wind energy penetration in the United States: a technical analysis of the energy resource, Black \& Veatch, B \& V project number 144864, 2007.

Archer, C. L. and Caldeira, K.: Global assessment of high-altitude wind power, IEEE T. Energy Conver., 2, 307-319, 2009.

Betz, A.: The maximum of theoretically available potential of wind by wind turbines (originally German), Z. Gesamte Turbinenwesen, Heft 26, 1920.

Blackman, C.: High-altitude winds: the greatest source of concentrated energy on Earth, http://news.stanford.edu/ news/2009/june24/high-altitude-winds-062309.html, last access: 16 June 2011, Stanford University News, 2009.

European Environment Agency: Europe's onshore and offshore wind energy potential, European Environment Agency, ISSN 1725-2237, 2009.

Energy Information Administration: International energy outlook 2010, United States Department of Energy - Energy Outlook Administration, 2010.

European Wind Energy Association: Oceans of opportunity: harnessing Europe's largest domestic energy resource, European Wind Energy Association, 2009.

Fraedrich, K., Jansen, H., Kirk, E., Luksch, U., and Lunkeit, F.: The planet simulator: towards a user friendly model, Meteorol. Z., 14, 299-304, 2005.
Gans, F., Miller, L. M., and Kleidon, A.: The problem of the second wind turbine - a note on a common but flawed wind power estimation method, Earth Syst. Dynam. Discuss., 1, 103-114, doi:10.5194/esdd-1-103-2010, 2010.

Garrett, C. and Cummins, P.: The efficiency of a turbine in a tidal channel, J. Fluid Mech., 588, 243-251, 2007.

Intergovernmental Panel on Climate Change: IPCC scoping meeting on renewable energy sources - proceedings, 2008.

Kirk-Davidoff, D. B.: Interactive comment on "Jet stream wind power as a renewable energy resource: little power, big impacts" by L. M. Miller et al., Earth Syst. Dynam. Discuss., 2, C240C242, 2011.

Kleidon, A.: Life, hierarchy, and the thermodynamic machinery of planet Earth, Phys. Life Rev., 7, 424-460, 2010.

Kleidon, A., Fraedrich, K., Kunz, T., and Lunkeit, F.: The atmospheric circulation and states of maximum entropy production, Geophys. Res. Lett., 30, 2223, doi:10.1029/2003GL018363, 2003.

Kleidon, A., Fraedrich, K., Kirk, E., and Lunkeit, F.: Maximum entropy production and the strength of the boundary layer exchange in an atmospheric general circulation model, Geophys. Res. Lett., 33, L06706, doi:10.1029/2005GL025373, 2006.

Lanchester, F. W.: A contribution to the theory of propulsion and the screw propeller, Trans. Inst. Naval Archit., LVII, 98-116, 1915.

Lansdorp, B. and Williams, P.: The laddermill - innovative wind energy from high altitudes in Holland and Australia. in: Global Windpower 06 conference, Adelaide, Australia. p. 1-14, 2006.

Li, L., Ingersoll, A. P., Jiang, X., Feldman, D., and Yung, Y. L.: Lorenz energy cycle of the global atmosphere based on reanalysis datasets, Geophys. Res. Lett., 34, L16813, doi:10.1029/2007GL029985, 2007.

Lorenz, E.: Available potential energy and the maintenance of the general circulation, Tellus, 2, 271-281, 1955.

Lunkeit, F., Bottinger, M., Fraedrich, K., Jansen, H., Kirk, E., Kleidon, A., and Luksch, U.: Planet simulator reference manual version 15.0, Meteorological Institute of the University of Hamburg, 2007.

Miller, L. M., Gans, F., and Kleidon, A.: Estimating maximum global land surface wind power extractability and associated climatic consequences, Earth Syst. Dynam., 2, 1-12, doi:10.5194/esd-2-1-2011, 2011.

Peixoto, J. P. and Oort, A. H.: Physics of climate, American Institute of Physics, Springer-Verlag, New York, USA, 1992.

Roberts, B. W., Shepard, D. H., Caldeira, K., Cannon, M. E., Eccles, D. G., Grenier, A. J., and Freidin, J. F.: Harnessing high-altitude wind power, IEEE T. Energy Conver., 22, 136-144, 2007.

US Department of Energy: $20 \%$ wind energy by 2030: increasing wind energy's contribution to US electricity supply, http://www. nrel.gov/docs/fy08osti/41869.pdf, last access: 11 March 2011, DOE/GO-102008-2567, 2008.

Vance, E.: High Hopes, Nature, 460, 564-566, 2009. 